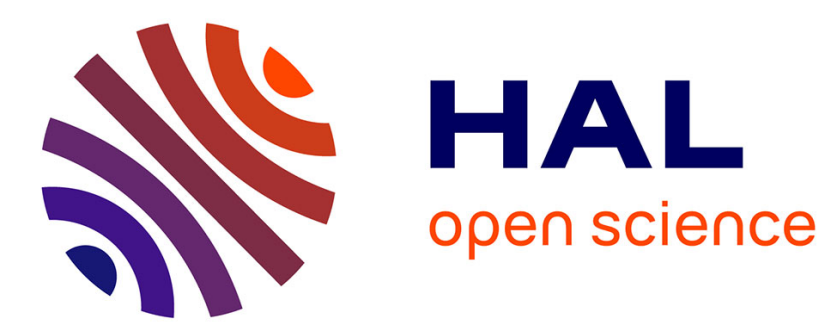

\title{
Hard x-ray single crystal bi-mirror
}

\author{
M. Lyubomirskiy, I. Snigireva, S. Kuznetsov, V. Yunkin, A. Snigirev, I. \\ Snigirev
}

\section{To cite this version:}

M. Lyubomirskiy, I. Snigireva, S. Kuznetsov, V. Yunkin, A. Snigirev, et al.. Hard x-ray single crystal bi-mirror. Optics Letters, 2015, 40 (10), pp.2205-2208. 10.1364/OL.40.002205 . hal-01572870

\section{HAL Id: hal-01572870 https://hal.science/hal-01572870}

Submitted on 8 Aug 2017

HAL is a multi-disciplinary open access archive for the deposit and dissemination of scientific research documents, whether they are published or not. The documents may come from teaching and research institutions in France or abroad, or from public or private research centers.
L'archive ouverte pluridisciplinaire HAL, est destinée au dépôt et à la diffusion de documents scientifiques de niveau recherche, publiés ou non, émanant des établissements d'enseignement et de recherche français ou étrangers, des laboratoires publics ou privés. 


\title{
Hard X-ray single crystal bi-mirror
}

\author{
M. Lyubomirskiy, ${ }^{1}$ I. Snigireva, ${ }^{1}$ S. Kuznetsov, ${ }^{2}$ V. Yunkin ${ }^{2}$ and A. Snigirev ${ }^{1 *}$ \\ ${ }^{I}$ ESRF, Grenoble, 38043, France \\ ${ }^{2} I M T$ RAS, 142432, Chernogolovka, Russia \\ *Corresponding author: snigireva@esrf.fr
}

Received Month X, XXXX; revised Month X, XXXX; accepted Month X, XXXX; posted Month X, XXXX (Doc. ID XXXXX); published Month X, XXXX

\begin{abstract}
We report a novel hard X-ray interferometer consisting of two parallel channels manufactured in a single Si crystal by means of microfabrication technology. The sidewall surfaces of the channels, similar to mirrors, scatter at very small incident angles, acting equivalently to narrow micrometer size slits as in the Young double-slit interferometer. Experimental tests of the interferometer were performed at the ESRF ID06 beamline in the energy range from $12 \mathrm{keV}$ to $16 \mathrm{keV}$. The interference patterns at different grazing incidence angles were recorded in the near- and far-field. Evaluation of the influence of the channel surface roughness on the visibility of interference fringes was performed. The proposed interferometer design allows the arrangement of mirrors at different split distances.

(030.0030) Coherence and statistical optics; (230.0230) Optical devices; (340.0340) X-ray optics.

http://dx.doi.org/00.0000/OL.00.000000
\end{abstract}

A number of experiments with double beam interference in the hard X-ray energy range were performed recently. The major experimental schemes [14] were taken from a visible optics wherein, in particular, special attention is given to schemes based on reflection geometry. The first X-ray interferometer based on reflection, known as a bi-mirror, consisted of two independent sequential mirrors placed on separate goniometers [5]. This set-up was used at grazing incidence angles below the critical angle for total external reflection. The advantage of such a technique is that the projected length of the mirrors to the observation plane is very small, making it equivalent to the Young's interferometer with narrow slits of micrometer size. Another approach, based on the differences between the critical reflection angles of two materials, was used to study the interference of reflected X-ray beams by thin gold stripes plated on the flat glass surface [6]. In the angular interval between the critical angles of substrate and coating, the ratio of the two materials reflection coefficients, $\left(\mathrm{R}_{\text {coat }} /\right.$ $\left.R_{\text {sub }}\right)$ is more than 100. While, outside this angular interval this ratio is much smaller and causes a weaker contrast of the interference fringes. In order to expand the angular interval of the interferometer operation, a setup with monolithic mirrors made from a $\mathrm{Si}$ crystal by removing a central part was proposed and used to measure the degree of spatial coherence of hard X-rays [7].

Modern microfabrication technologies enable profiling $\mathrm{Si}$ crystals creating significantly deep and vertical trenches [8] with the quality of the etched sidewall surfaces which allows the observation of X-ray total external reflection. In the present paper we propose a new type of bi-mirror interferometer consisting of two parallel reflecting surfaces made in a Si single crystal.

The schematic design of bi-mirror interferometer is shown in Fig. 1(a). The novelty of our interferometer is that the reflecting surfaces are arranged vertically one above the other, while in former interferometers they are placed sequentially one after another. This design allows the reduction of the vertical gap $D$ between the mirrors, permitting to improve the contrast of the interference pattern in the case of poor spatial coherence, and furthermore to observe the far-field interference pattern at much shorter distances. The interferometer was manufactured using a process involving electron beam lithography and deep etching into silicon [8]. A scanning electron microscope (SEM) image of the bi-mirror interferometer is shown in the Fig. 1(b). The interferometer consists of two identical vertically arranged mirror channels made in the silicon crystal. The total length of the channels $b$ is $20 \mathrm{~mm}$, and they are 10 $\mu \mathrm{m}$ wide. The thickness of the Si bars or slabs is $10 \mu \mathrm{m}$, so the mirror split distance $D$ is $20 \mu \mathrm{m}$.

At grazing incidence angles the vertical sidewall surface of channels resembles a mirror. The X-ray beam reflects from the sidewalls and at some distance the diffracted cones overlap giving rise the interference in this region. According to the Huygens-Fresnel principle, mirrors at grazing angles can be considered as secondary radiation sources. The size of each secondary source is equal to the slit width of the equivalent Young interferometer and can be modified by changing the angle $\theta$ between the incident beam and a mirror surface. So we have "virtual" slits with size $d$ as a function of the grazing angle $\theta$, namely, as a projection of the mirror length $d(\theta)=b \sin \theta$, where $b$ is the total length of the mirror surface. Since the distance between "virtual" slits is weakly affected by the grazing incidence angle which is less than $0.1^{\circ}$, we can exclude it from the consideration and assume the gap between slits $D$ is constant. In comparison with the sequential arrangement of mirrors, in which $D$ changes with the angle of incidence, this is an advantage. Similar to the Young double slit scheme, the split distance $D$ of the bimirror should be smaller than a spatial coherence length of the incoming beam defined as $l_{c o h}=\lambda^{*} L_{1} / s$, where $\lambda$ is the radiation wavelength, $s$ is the effective source size (FWHM), and $L_{1}$ is the source-to-interferometer distance. 
The intensity distribution in the far-field diffraction pattern is formed as the result of overlap between waves diffracted on two virtual slits $[9,10]$ :

$$
I(x)=I_{0} \operatorname{sinc}^{2}\left(k_{d} x\right) \times\left[1+M \cos \left(k_{D} x\right)\right]+I_{b},
$$

where

$$
k_{d}=\frac{2 \pi d}{\lambda L_{2}}, k_{D}=\frac{2 \pi D}{\lambda L_{2}}, M=\operatorname{sinc}\left(\pi D s / \lambda L_{1}\right) \text {, }
$$

Here $L_{2}$ is the distance to the observation plane, $d$ is the slit size, $D$ is the distance between slits, $s$ is the source size, and $I_{b}$ is the background intensity. The observed intensity pattern can be characterized by the quantitative parameter of interference fringe visibility $V$.

$$
V=\frac{I_{\max }-I_{\min }}{I_{\max }+I_{\min }} \times 100 \%
$$

where $I_{\max }$ and $I_{\min }$ are values of the irradiances corresponding to the maximum and adjacent minimum on the interference fringe pattern, respectively. From (1) it follows that for coherent radiation $\left(I_{b}=0\right)$ maximum and minimum adjacent intensities are:

$$
\begin{aligned}
& I_{\text {max }}=I_{0}(1+M) \\
& I_{\text {min }}=I_{0} \operatorname{sinc}^{2}(\pi d / D)(1-M)
\end{aligned}
$$

As for the Young double slit, the visibility is primarily a function of the source size. We have calculated the fringe visibility versus the source size $s$ varied from $25 \mu \mathrm{m}$ to $300 \mu \mathrm{m}$. For calculations the following parameters were used: $d / D=0.1, D=20 \mu \mathrm{m}, L_{1}=50 \mathrm{~m}, \lambda=1 \AA$ (photon energy $12 \mathrm{keV}$ ). The fringe visibility is close to $100 \%$ for a source size of $25 \mu \mathrm{m}$, typical for undulator beamlines at the ESRF, and visibility is more than $50 \%$ for the source size up to $150 \mu \mathrm{m}$.

The experimental test of bi-mirror interferometer has been performed at the Micro Optics Test Bench (MOTB) at the ESRF ID6 beamline. A liquid nitrogen cooled Si111 double crystal, fixed exit monochromator was used to adjust X-ray energy in the range $12-16 \mathrm{keV}$. The source size was measured by a B-fiber interferometry technique [11], and during the bi-mirror interferometer tests was on the order of 40 microns. The interferometer was mounted on the stage with all necessary rotation and translation movements at a distance of 56 meters from the source (see Fig.1.). At this distance, the spatial coherence length is about $100 \mu \mathrm{m}, 5$ times larger than bi-mirror separation so the irradiation may be considered coherent. The measurement of the interference was performed with a high resolution X-ray CCD camera equipped with a fluorescence screen and an optical objective which giving $1.3 \mu \mathrm{m}$ spatial resolution. The measurement of interference patterns was performed at different X-ray incidence angles $\theta$, achieved by rotating the interferometer. The typical exposure time varied between 10 seconds and 1 minute depending on the incidence angle and observation distance, the storage ring mode was $7 / 8+1$ with a current approximately $200 \mathrm{~mA}$.
To characterize the interferometer in the near-field, 12 $\mathrm{keV}$ was chosen and CCD was placed downstream from the interferometer at the distance $L_{2}=1.5 \mathrm{~m}$. The observed interference pattern and intensity variation obtained for the line through the center of the fringe pattern measured at grazing incidence angle $\theta=0.004^{\circ}$ is shown in Fig. $2(\mathrm{a}, \mathrm{b})$. The cross section of interference patterns measured at grazing incidence angle $\theta=0.007^{\circ}$ is presented in Fig. 2(c). The quantitative evaluation of interference patterns was performed by the visibility parameter $V$, calculated in the center of the pattern in a region of maximum overlap of diffracted beams. These incidence angles correspond to the mirror projection or "virtual" slit sizes of $d=1.4 \mu \mathrm{m}$ and $d=2.5 \mu \mathrm{m}$, respectively.

The interference fringe visibility corresponding to the lower angle $\theta=0.004^{\circ}$ is $58 \%$ whereas for the angle $\theta=0.007^{\circ}$ the visibility is $54.5 \%$. Increasing the incident angle of the primary beam leads to reduction of the mirror diffraction cones resulting in the narrowing of the overlapping area which in turn reduces the contrast. It is evident that in order to obtain reliable experimental data, which can be easily interpreted, it is necessary to measure the interference pattern in the far-field.

Taking into account the beamline characteristics such as energy range, available distances, and efficiency of the CCD camera, the far field tests of the bi-mirror interferometer were performed at $16 \mathrm{keV}$ photon energy (0.77 $\AA$ wavelength) and at mirror-to-detector distance $L_{2}=15$. It can be easily estimated that for this photon energy and $20 \mu \mathrm{m}$ bi-mirror separation the far-field observation distance (the region of Fraunhofer diffraction) must be more than $L=D^{2} / \lambda \approx 5 \mathrm{~m}$.

The far field intensity variations obtained for the line through the center of the fringe pattern recorded at angles of incidence $\theta=0.009^{\circ}$ and $\theta=0.021^{\circ}$ are depicted in Fig. $3(\mathrm{a}, \mathrm{b})$. Contrary to near field measurements, the maximum measured visibility of $86 \%$ corresponds to the higher incident angle, a clear demonstration that far field conditions are fulfilled. Fig. 3(c) presents the dependence of fringe visibility on the incidence angle. Depending on the incident angle, the contrast of the fringes varied within $10 \%$, steadily increasing with incident angle. However, we would like to stress that the calculated fringe visibility for a $40 \mu \mathrm{m}$ source is about $95 \%$ and the changing of the incidence angle does not affect significantly the contrast (for example, a doubling of the grazing incidence angle from $0.01^{\circ}$ to $0.02^{\circ}$ increases the fringe visibility from $93 \%$ to $95 \%$ ). The formally estimated source size from the bi-mirror fringe visibility at grazing incidence angle $\theta=0.009^{\circ}$ is $s=78 \mu \mathrm{m}$ while for angle $\theta=0.021^{\circ}$ is $S=55 \mu \mathrm{m}$ that is essentially closer to the result of the B-fiber $s=40 \mu \mathrm{m}$.

The reduction of the fringe contrast and its pronounced angular dependence can be caused by diffuse scattering caused by the roughness of the etched surface. In order to estimate this effect, we made an evaluation of the surface roughness from the visibility of interference fringes.

Our experiment is carried out under grazing incidence and reflection conditions where the angles of incidence are much less than the critical angle of total reflection $\theta_{c}$, which for $\mathrm{Si}$ is between $0.15^{\circ}$ and $0.11^{\circ}$ in the photon 
energy range $10-16 \mathrm{keV}$. In this case, the specularly reflected component of the scattering should be analyzed as the Fresnel reflectivity. Roughness reduces the reflected amplitude, therefore it is necessary to consider its influence in the Fresnel equations introducing the damping factor. To take into account the contribution of the roughness in the Fresnel reflectivity, the well-known Gaussian damping, given by Rayleigh, is the most widely used, although it does not fully describe the measured intensity of the reflected of X-rays [12,13]. If a surface roughness is small and if a Gaussian distribution of roughness heights is assumed, the specular intensity can be written as

$$
I=I_{0} \exp \left(-4 k^{2} \theta^{2} \sigma^{2}\right)
$$

where $\sigma$ is the root-mean-square (rms) surface roughness, $\theta$ is the grazing angle of the reflection, and $k$ is the wave vector of the radiation equal to $2 \pi / \lambda$. In order to obtain a more appropriate description of the reflectivity in the vicinity of the critical angle an improved expression for the damping factor, where it is assumed the loss of the coherence of the X-ray wave on the rough surface, was proposed in [13]. However, this approach is not suitable for grazing angles of much less than the critical angle.

Using (5) the irradiances $I^{T} \max$ and $I^{T}$ min corresponding to the maximum and adjacent minimum on the interference pattern (Eq. 3 and 4) should be rewritten as

$$
\begin{aligned}
& I_{\max }^{T}=I_{0} \exp \left(-4 k^{2} \theta^{2} \sigma^{2}\right)(1+M) \\
& I_{\min }^{T}=I_{0} \exp \left(-4 k^{2} \theta^{2} \sigma^{2}\right) \times \operatorname{sinc}^{2}\left(\pi \frac{d}{D}\right) \times(1-M)
\end{aligned}
$$

Let us assume that all the background intensity consists of the diffuse part of the reflected beam (we neglect the absorption), i.e.

$$
I_{b}=I_{0}\left[1-\exp \left(-4 k^{2} \theta^{2} \sigma^{2}\right)\right]
$$

and that the visibility $V_{\exp }$, calculated from the experimental data of intensity cross-sections, should be expressed theoretically by taking into account background intensity:

$$
V_{\exp }=\frac{I_{\max }^{T}-I_{\min }^{T}}{I_{\max }^{T}+I_{\min }^{T}+2 I_{b}}
$$

If a surface roughness and grazing angle of the reflection are very small then we can replace the exponent by the sum $\exp \left(4 k^{2} \theta^{2} \sigma^{2}\right) \approx 1+4 k^{2} \theta^{2} \sigma^{2}$, and from expressions (6)-(9) we obtain the value of the dumping factor and hence the rms surface roughness as

$$
4 k^{2} \theta^{2} \sigma^{2}=\frac{1}{2}\left\{(1+M)+(1-M) \operatorname{sinc}^{2}\left(\pi \frac{d}{D}\right)\right\}\left[\frac{V_{T}}{V_{\text {exp }}}-1\right]_{(10)}
$$

where $V_{T}$ is the theoretical visibility calculated by Eq. (2$4)$.
For example, a measured visibility of $87 \%$ at grazing incidence angle $0.021^{\circ}$ gives an rms surface roughness $\sigma=55 \AA$, whereas for a measured visibility of $77 \%$ at grazing incidence angle $0.009^{\circ}$ corresponds to an $\mathrm{rms}$ surface roughness $\sigma=185 \AA$.

We notice that from our estimations the influence of the surface roughness on the visibility at very small incident angles is more pronounced and results in a low value of fringe contrast. Such a large difference in the roughness results from the limitation of the proposed roughness evaluation model. It should be noted that the surface after the reactive-ion etching process used to produce $\mathrm{Si}$ trenches has a very complex topography [8] resulting in a strongly varying power spectral density (PSD). We do not exclude that the spatial frequencies probed are diverse enough that very different integrals of PSD could result.

The bi-mirror interferometer for hard X-rays was designed and manufactured in a $\mathrm{Si}$ crystal using microfabrication planar technology. This new design allowed the creation of reflecting surfaces at any split distance. The close arrangement of mirrors makes it possible to observe high contrast interference pattern for sources with poor spatial coherence such as X-ray laboratory sources. The bi-mirror was experimentally tested in the near and far filed conditions. The quality of the etched sidewall surfaces is sufficient to register interference patterns with fringe contrast of $86 \%$. The decrease of fringe visibility, especially noticeable at small grazing angles, results from the diffuse scattering due to the surface roughness of mirrors. A model, based on rather simple and approximate assumptions, was considered for the evaluation the influence of the channel surface roughness on the visibility of interference fringes. A roughness of the etched surface smaller than $185 \AA$ rms was estimated based on the collected data and it is in a good agreement with previous results [8].

This bi-mirror interferometer is implemented on a multifunctional X-ray silicon chip which includes other optical elements such as compound parabolic refractive lenses [14], bi-lens [3], and multi-lens interferometers based on refractive lenses [15]. Microfabrication technology, used in manufacturing of the universal silicon chip, ensures that the bi-mirror interferometer reflective surfaces, made simultaneously with the compound refractive parabolic lenses, are identical in surface roughness. Therefore, the assessment of roughness obtained from bi-mirror interferometry data will be valuable for the evaluation of the focusing properties of the compound refractive lenses and, obviously, for improvement of the technological processes.

The authors are grateful to C. Detlefs and P. Wattecamps for their help and support during the experiments at ID 06 beamline. The authors thank O. Konovalov for the helpful discussions. The work was supported by the Ministry of Science and Education of Russian Federation grants № 14.Y26.31.0002, № 02.G25.31.0086. 
1. A. R. Lang, and A. P. W. Makepeace, J. Synchrotron Radiat. 6, 59-61 (1999).

2. W. Leitenberger, S. M. Kuznetsov, and A. Snigirev, Opt. Commun. 191, 91-96 (2001).

3. A. Snigirev, I. Snigireva, V. Kohn, V. Yunkin, S. Kuznetsov, M. B. Grigoriev, T. Roth, G. Vaughan, and C. Detlefs, Phys. Rev. Lett. 103, 064801 (2009).

4. C. Chang, P. Naulleau, E. Anderson, and D. Attwood, Nucl. Instr. Meth. Phys. Res. Sect. A 467-468, Part 2, 913-916 (2001).

5. K. Fezzaa, F. Comin, S. Marchesini, R. Coïsson, and M. Belakhovsky, J. Xray Sci. Technol. 7, 12-23 (1997).

6. S. Aoki, N. Watanabe, T. Ohigashi, H. Yokosuka, Y. Suzuki, A. Takeuchi, and H. Takano, Jpn. J. Appl. Phys. 44, 417 (2005).

7. W. Leitenberger, and U. Pietsch, J. Synchrotron Radiat. 14, 196-203 (2007).

8. A. Snigirev, I. Snigireva, M. Grigoriev, V. Yunkin, M. Di Michiel, S. Kuznetsov, and G. Vaughan, Proc. SPIE 6705, 670506 (2007).

9. J. W. Goodman, Introduction to Fourier Optics (McGraw-Hill Book Company, 1968).

10. M. Françon, Optical Interferometry (Academic Press, 1966).

11. V. Kohn, I. Snigireva, and A. Snigirev, Opt. Commun. 198, 293-309 (2001).

12. S. K. Sinha, E. B. Sirota, S. Garoff, and H. B. Stanley, Phys. Rev. B 38, 2297-2311 (1988).

13. F. Stanglmeier, B. Lengeler, W. Weber, H. Gobel, and M. Schuster, Acta Crystallogr. Sect. A 48, 626-639 (1992).

14. A. Snigirev, V. Kohn, I. Snigireva, and B. Lengeler, Nature 384, 49-51 (1996).

15. A. Snigirev, I. Snigireva, M. Lyubomirskiy, V. Kohn, V. Yunkin, and S. Kuznetsov, Opt. Express 22, 25842-25852 (2014).
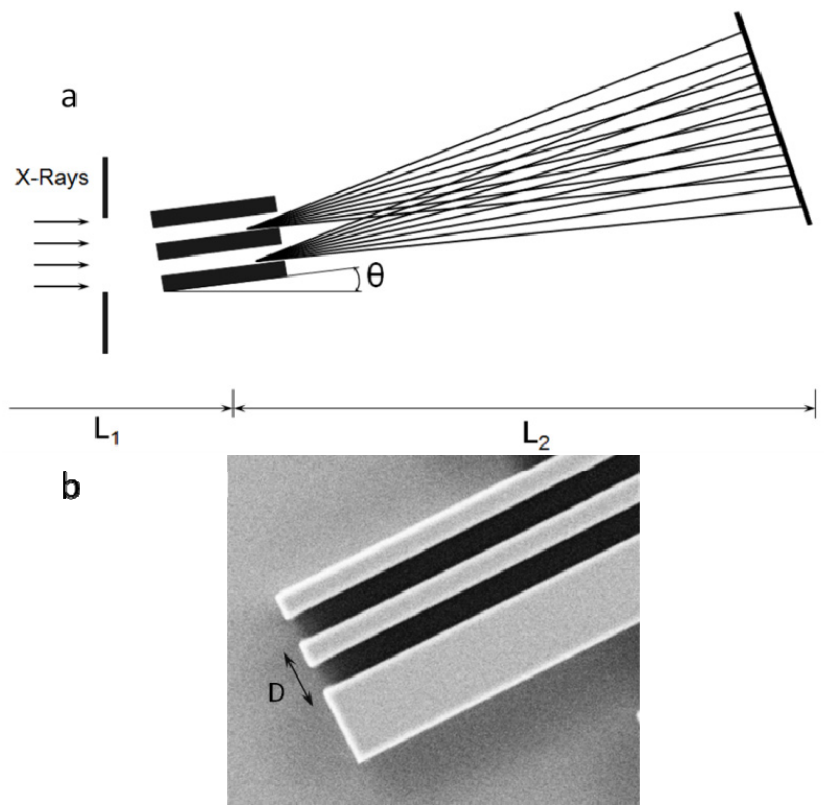

Fig.1. Schematic design of bi-mirror interferometer (a). SEM image of bi-mirror channels (b).

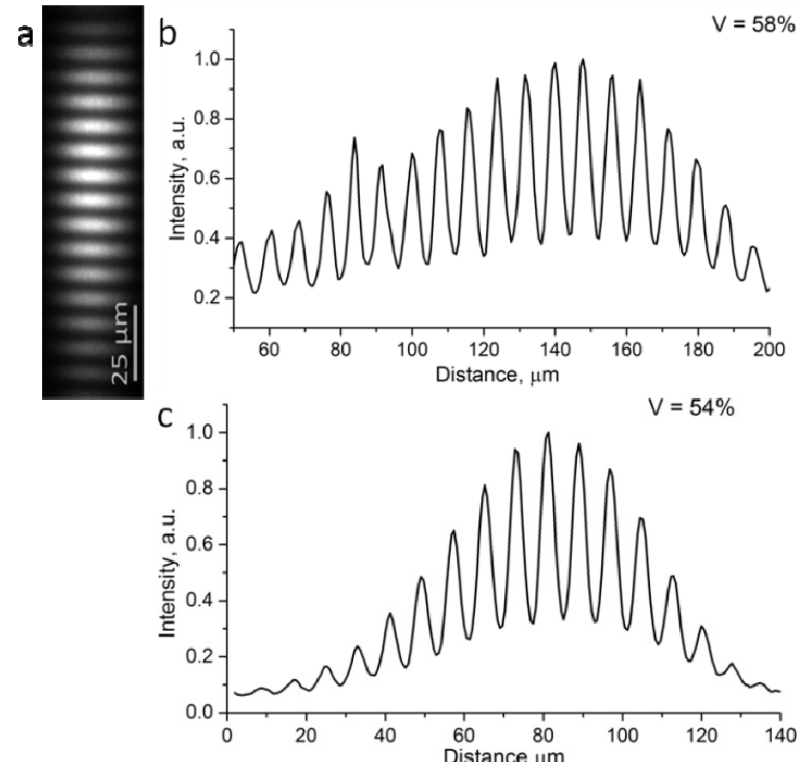

Fig.2. The interference pattern (a) and the intensity variation obtained for the line through fringe center (b) recorded at incidence angle $0.004^{\circ}$. The intensity variations for the interference pattern registered at incidence angle $0.007^{\circ}$ (c).
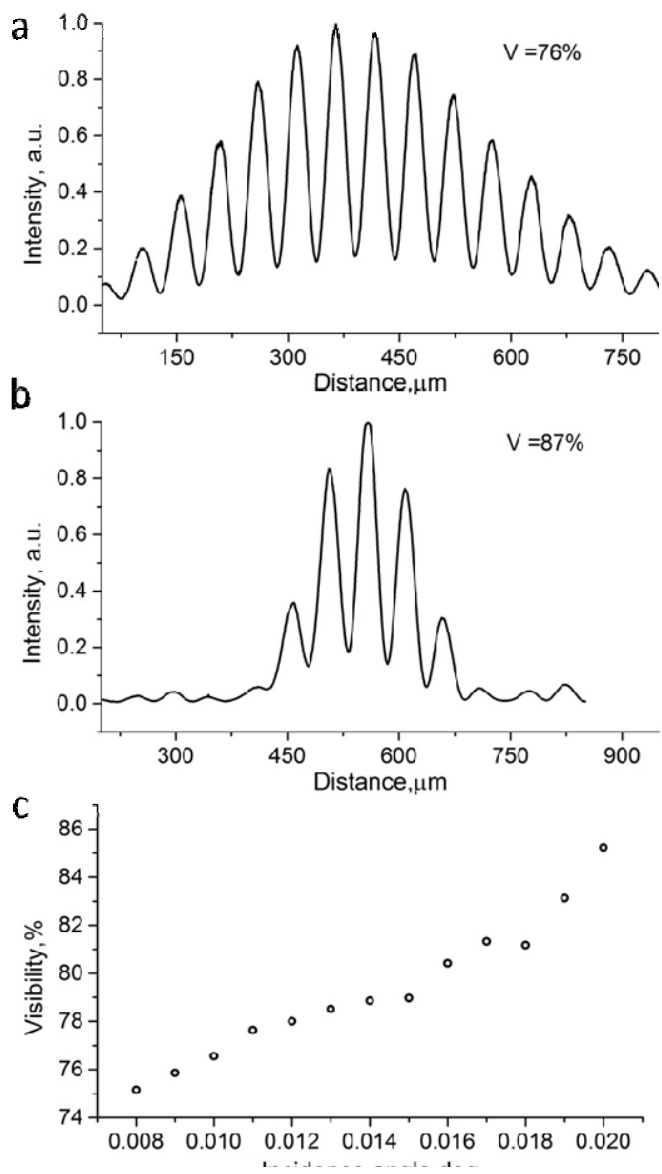

Fig.3. Interference pattern vertical cross section for incidence angle $0.009^{\circ}$ (a), cross section of the interference pattern for incidence angle $0.021^{\circ}(\mathrm{b})$ and angular dependence of the fringe visibility (c). 
1. A.R. Lang, A.P.W. Makepeace, Production of synchrotron X-ray biprism interference patterns with

2. W. Leitenberger, S.M. Kuznetsov, A. Snigirev, Interferometric measurements with hard X-rays using a double slit, Opt. Commun. 191, 91-96 (2001).

3. A. Snigirev, I. Snigireva, V. Kohn, V. Yunkin, S. Kuznetsov, M.B. Grigoriev, T. Roth, G. Vaughan, C. Detlefs, X-Ray Nanointerferometer Based on Si Refractive Bilenses, Phys. Rev. Lett. 103, 064801 (2009).

4. C. Chang, P. Naulleau, E. Anderson, D. Attwood, Spatial coherence properties of undulator radiation based on Thompson-Wolf two-pinhole measurement, Nucl. Instr. Meth. Phys. Res. Sect. A 467-468 (2), 913-916 (2001).

5. K. Fezzaa, F. Comin, S. Marchesini, R. Coïsson, M. Belakhovsky, X-Ray Interferometry at ESRF Using Two Coherent Beams from Fresnel Mirrors, Xray Sci. Technol. 7, 12-23 (1997).

6. S. Aoki, N. Watanabe, T. Ohigashi, H. Yokosuka, Y. Suzuki, A. Takeuchi, H. Takano, Production of Reflection Point Sources for Hard X-Ray Gabor Holography, Jpn. J. Appl. Phys. 44, 417 (2005).

7. W. Leitenberger, U. Pietsch, A monolithic Fresnel bimirror for hard X-rays and its application for coherence measurements, J. Synchrotron Radiat. 14, 196-203 (2007).

8. A. Snigirev, I. Snigireva, M. Grigoriev, V. Yunkin, M. Di Michiel, S. Kuznetsov, G. Vaughan, Silicon planar lenses for high-energy x-ray nanofocusing, Proc. SPIE 6705, 670506 (2007).

9. J.W. Goodman, Introduction to Fourier Optics, McGraw-Hill Book Company, 1968.

10. M. Françon, Optical Interferometry, Academic Press, 1966.

11. V. Kohn, I. Snigireva, A. Snigirev, Interferometric characterization of spatial coherence of high energy synchrotron X-rays, Opt. Commun. 198, 293-309 (2001).

12. S.K. Sinha, E.B. Sirota, S. Garoff, H.B. Stanley, Xray and neutron scattering from rough surfaces, Phys. Rev. B 38, 2297-2311 (1988).

13. F. Stanglmeier, B. Lengeler, W. Weber, H. Gobel, M. Schuster, Determination of the dispersive correction $\mathrm{f}^{\prime}(\mathrm{E})$ to the atomic form factor from X-ray reflection, Acta Crystallogr. Sect. A 48, 626-639 (1992).

14. A. Snigirev, V. Kohn, I. Snigireva, B. Lengeler, A compound refractive lens for focusing high-energy X-rays, Nature 384, 49-51 (1996).

15. A. Snigirev, I. Snigireva, M. Lyubomirskiy, V. Kohn, V. Yunkin, S. Kuznetsov, X-ray multilens interferometer based on Si refractive lenses, Opt. Express 22, 2584225852 (2014). 